\title{
Using Protein Folding Rates to Test Protein FOLDING THEORIES
}

Blake Gillespie ${ }_{1,3}$ and Kevin W. Plaxco ${ }_{1,2}$

1Department of Chemistry and Biochemistry, ${ }_{2}$ Interdepartmental Program in Biomolecular Science and Engineering, University of California, Santa Barbara, Santa Barbara, California 93106; e-mail: kwp@chem.ucsb.edu ${ }_{3}$ Chemistry Program, California State University Channel Islands, Camarillo, California 93012; email: blake.gillespie@csuci.edu

Key Words simulation, nucleation, topomer search

Abstract The fastest simple, kinetically two-state protein folds a million times more rapidly than the slowest. Here we review many recent theories of protein folding kinetics in terms of their ability to qualitatively rationalize, if not quantitatively predict, this fundamental experimental observation.

\section{INTRODUCTION: TWO-STATE FOLDING RATES AS AN EXPERIMENTAL BENCHMARK}

Simple, single-domain proteins typically fold via a process that lacks well-populated intermediates [reviewed in (1)]. Despite the potential simplification afforded by the absence of kinetic intermediates, reported two-state folding rates span a remarkable six order of magnitude range (Figure 1) $(2,3)$. When coupled with the appealing simplicity of two-state behavior, the broad range of two-state rates provides a potentially straightforward and quantitative opportunity to test theories of the folding process.

Theoretical models of protein folding kinetics can, admittedly somewhat artificially, be grouped into two broad classes. Perhaps the more prominent class consists of theories emerging from observations of the simulated folding of simple on- and off-lattice models in silico. The second class consists of theoretical models emerging from the experimental observation of protein folding in vitro. In this article, we broadly review many recent simulation- and experiment based theories of folding kinetics and critically evaluate these theories in terms of their ability to qualitatively rationalize, if not quantitatively predict, the vast range of folding rates observed for two-state proteins. 


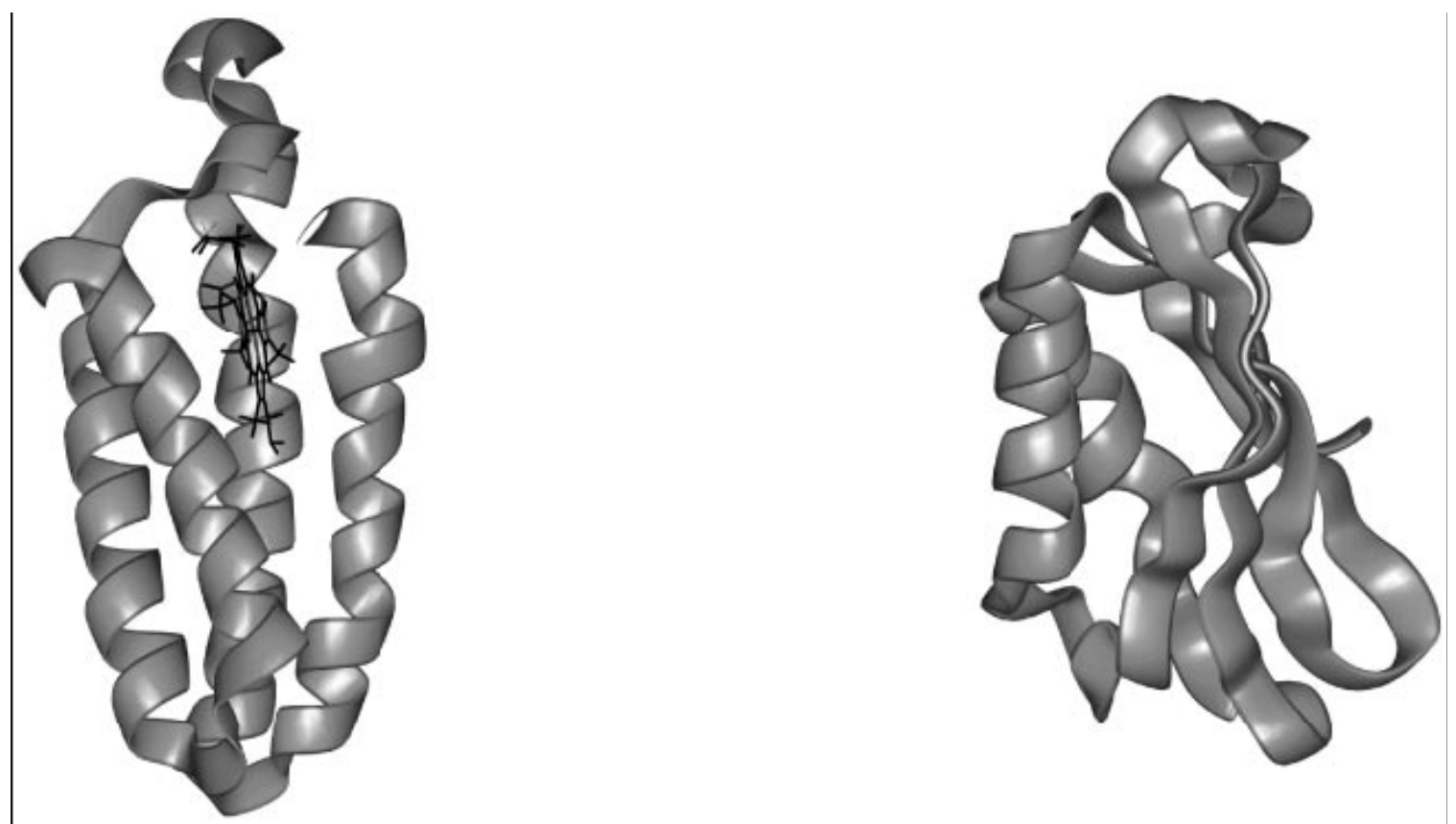

Figure 1 The simple, single-domain proteins cytochrome $b_{562}$ and muscle acylphosphatase are of similar size and stability, yet the former folds in microseconds, and the latter folds in seconds $(2,3)$. Here we critically review the ability of various theories of protein folding to rationalize qualitatively, if not predict quantitatively, this six order of magnitude range of rates.

\section{SIMULATION-DERIVED THEORIES OF FOLDING}

Perhaps the majority of contemporary theories of protein folding kinetics are based on observations of the simulated folding of simple, computational models. In an ideal world, the relevant simulations would entail a level of detail commensurate with the complex, atomistic structure of a fully solvated polypeptide. In reality, however, the simulation of protein folding in atomistic detail has proven computationally overwhelming. The difficulty is twofold. First, computational times scale strongly with the number of atoms, and even the smallest proteins are composed of thousands of atoms solvated by thousands of water molecules. Second, even the most rapidly folding proteins fold extremely slowly relative to the femtosecond time step of fully detailed molecular dynamics simulations [for a recent, partial solution to this dilemma, see Pande and coworkers folding@home project (4)]. Faced with this computational obstacle, the large majority of the theoretical literature in protein folding has been based on observations not of fully detailed protein models but on highly simplified representations of the polypeptide chain. Lattice polymers, perhaps the most popular computational model, simplify the description of the polypeptide chain by distilling each amino acid into a single bead and simplify folding dynamics by limiting the moves of each bead to hops between discrete points on a coarse lattice.

Although lattice polymers and many off-lattice computational models are highly simplified, they capture many of the potentially relevant aspects of real proteins. For example, lattice polymers, similar to proteins, are sequence-specific heteropolymers that can encode a unique native fold, and though the coarse lattice significantly reduces the entropy of the unfolded state, its entropy is still sufficiently large that folding would be 
slow were it a fully random search process. Similarly, some lattice polymer sequences surmount this entropic barrier (the Levinthal Paradox) much more efficiently than others, and thus lattice polymers exhibit a wide range of folding rates. To date a major goal of computational folding studies has been the identification of the equilibrium properties that uniquely identify those rare lattice polymer sequences that fold rapidly, under the assumption that similar behavior will underlie the rapid folding of real proteins. The criteria predicted to distinguish between the rapidly and slowly folding lattice polymer sequences thus provide a clear opportunity for evaluating the correspondence between theory and experiment in protein folding.

In the last decade alone, more than 700 papers on the folding kinetics of simple onand off-lattice protein models have appeared in the literature. Although it is impossible to accurately distill such a large, diverse, and often conflicting literature into a few brief conclusions, much of this literature can be classified by the criterion that is predicted to separate rapidly folding sequences from slowly folding sequences. Here we briefly describe the three major criteria that have been suggested as potential determinants of the relative folding rates of simplified computational models and review the experimental literature for clues as to whether similar criteria are responsible for the broad range of rates observed for the folding of two-state proteins.

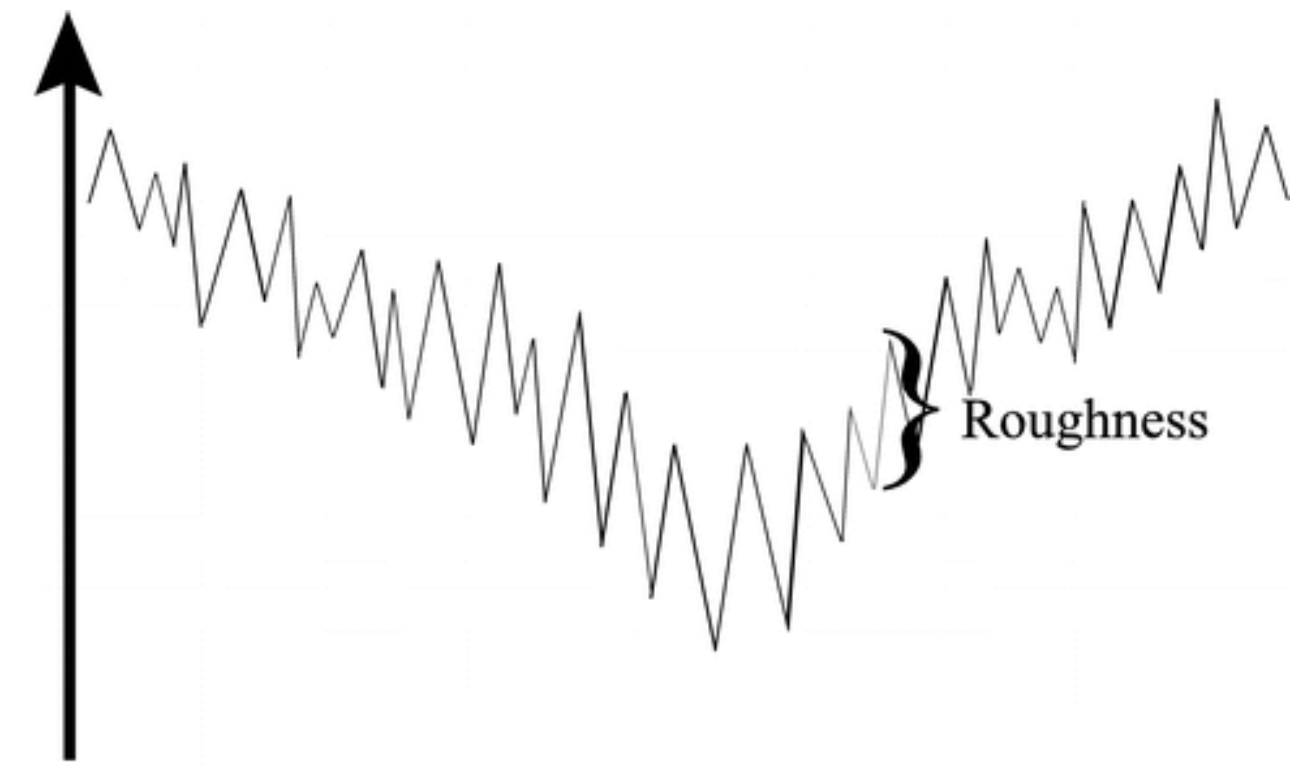

\section{Energy Reaction coordinate}

Figure 2 Rapid folding will occur when the energy of each unfolded or partially folded conformation decreases more or less monotonically [along some simple, but often difficult to define, order parameter(s)] as conformations become more native-like $(5,6)$. Roughness on this energy landscape can produce complex, stretched-exponential kinetics in lattice polymers (9) and may also account for part of the broad range of observed two-state folding rates.

\section{Smooth Energy Landscapes}


A large body of theoretical work suggests that the rapid folding of lattice polymers $(5$, 6 ) and simplified off-lattice polymers $(7,8)$ is associated with smooth, funnel-shaped energy landscapes (Figure 2). That is, rapid folding will occur when the energy of each unfolded or partially folded conformation decreases more or less monotonically as conformations become more native-like along some reaction coordinate(s). If this energetic guidance does not occur, or if the landscape is rough (contains local minima deeper than a few $k_{B} T$ that act as traps), folding becomes glassy, dominated by multiple kinetic traps, and slows dramatically. Differences in the roughness of the energy landscape can lead to orders of magnitude changes in the folding rates of simplified computational models (9).

How can we determine if variations in energy-landscape roughness contribute significantly to the relative folding rates of small proteins? If energetic roughness dominates the energy landscape, slow, nonsingle-exponential kinetics will be observed $(5,9,10)$. That is, at temperatures at which $k_{B} T$ is small relative to the myriad kinetic barriers on a rough landscape (near the so-called glass transition temperature, $T_{g}$ ), the myriad of local kinetic barriers will begin to retard the folding process, switching the kinetics from single exponential $(h=1)$ to a slower, stretched exponential $(h>1)$ :

$$
S(t)=S_{n}+A_{0} \exp \left(-\left(k_{f} t\right)^{1 / h}\right)
$$

where $k f$ is the folding rate, $A_{0}$ denotes the amplitude change upon folding, and $S_{n}$ and $S_{(t)}$ respectively denote the signal of the native state and that observed at time $t(5,8-10)$. We can determine the extent to which roughness defines relative folding rates in the laboratory by employing this metric to measure the relative energy-landscape roughness of both rapidly and slowly folding proteins.

Do differences in energy-landscape roughness account for a significant fraction of the $1,000,000$-fold range of rates observed for two-state protein folding? Although indications of landscape roughness have been reported for the single-domain protein ubiquitin [(11), but see commentary in (12)] at low temperatures, energetic roughness does not generally appear to account for the vast range of folding rates observed under more physiological conditions. Once the complication of proline isomerization is taken into account, the large majority of simple, single domain proteins appear to fold with single exponential kinetics $(1,11,12)$. Examples include the folding of the 62-residue protein L, which appears perfectly single exponential down to the experimental limit of $15^{\circ} \mathrm{C}(12)$, and the pI3k-SH3 domain, which also exhibits a smooth energy landscape despite being the second most slowly folding two-state protein reported to date (13) (Figure 3). It thus appears that although theory is correct in associating smooth energy landscapes with the rapid folding of naturally occurring proteins (5-8), differences in the roughness of the energy landscape do not play a significant role in defining the six order of magnitude range of observed two-state folding rates. 


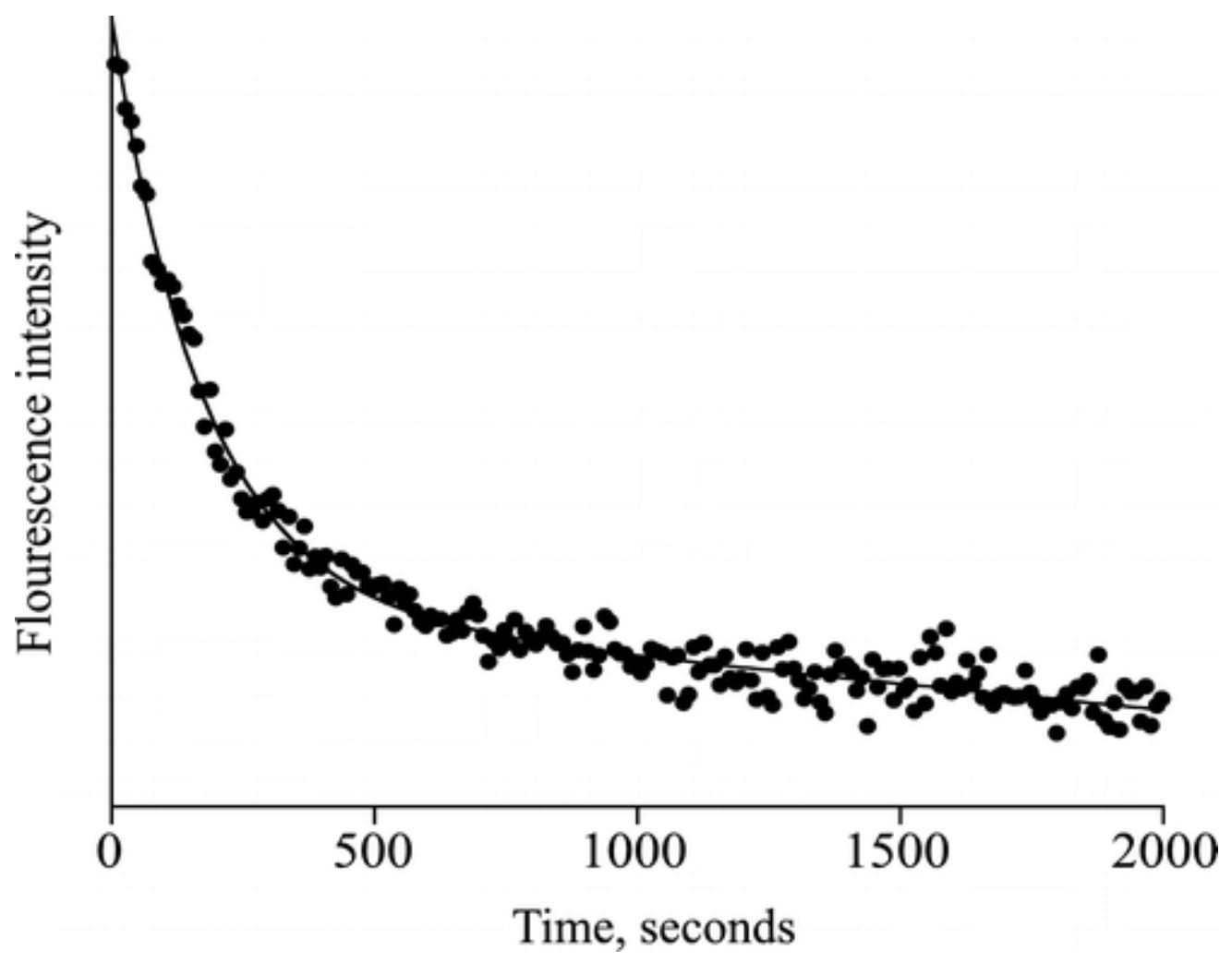

Figure 3 The folding energy landscapes of even the most slowly folding single-domain proteins are exceptionally smooth. Shown here is the refolding of the pI3 k-SH3 domain, one of the most slowly folding of all two-state proteins (13), after folding is initiated by rapid dilution at $5^{\circ} \mathrm{C}$. There is no statistically significant evidence of deviation from the fitted single-exponential kinetics (B. Gillespie and K.W. Plaxco, unpublished observations), even at this low temperature, where $k_{B} T$ is reduced and the effects of landscape roughness enhanced. It appears that energetic roughness plays no significant role in slowing the folding of even the most slowly folding two-state proteins (12).

\section{The Energy Gap Hypothesis}

Karplus and coworkers have reported that a "necessary and sufficient criterion to ensure [rapid] folding is that the ground state be a pronounced energy minimum (14)" relative to all other maximally compact states (Figure 4). More recently these and other researchers have identified a number of related measures of energetic gap between the ground state and other maximally compact states. These include the $\mathrm{Z}$ score, which is a measure of the statistical significance of the size of the gap between the energy of the native state and the mean energy of all other maximally compact states (15). This measure of the energy gap is reported to correlate significantly with the folding rates of a number of simple lattice polymers (16) and may account for the vast dispersion observed in the folding rates of real proteins.

Unfortunately, we cannot measure the size of the energy gap experimentally, and thus, we cannot directly determine whether differences in the energy gap account for the wide range of observed protein folding rates. The difficulty is that, as demonstrated by both solution-phase and crystallographic structural studies, the vast majority of proteins populate only a single maximally compact state, the native state, and therefore, the energy gap between the ground state and all other maximally compact states is too large 
to measure. An indirect experimental test of the energy gap hypothesis may be provided, however, by the empirical observation that, for some lattice polymer models, the melting temperature ( $T_{m}$, often denoted $T_{f}$ in the theoretical literature) is correlated with the magnitude of the energy gap. This leads to the (indirect, empirically based) prediction that folding rates should also correlate with $T_{m}(16)$. To the extent that this has been monitored experimentally, however, it appears that folding rates are generally uncorrelated with $T_{m}$. For example, although folding rates may be modestly correlated with $T_{m}$ across a set of mutants of a single protein (A. Fersht, personal communication), a survey of the experimental literature suggests that $T_{m}$ is effectively completely uncorrelated with folding rates across a set of nonhomologous proteins (Figure 5). Even for sets of closely related proteins, the correlation between $T_{m}$ and folding rates is often nonexistent. A set of homeodomain sequences provides an extreme example: Despite $T_{m}$ ranging from $54^{\circ} \mathrm{C}$ to $116^{\circ} \mathrm{C}$, the folding rates of the three characterized homeodomains are effectively within error of one another $(12,17$; B. Gillespie and K.W. Plaxco, unpublished data). Similarly, there is no correlation between $T_{m}$ and folding rates across mesophile-, thermophile-, and hyperthermophile-derived cold shock proteins (18). Thus, although theory is correct in associating a large energy gap with the rapid folding of naturally occurring proteins, it appears that there is no evidence in favor of the hypothesis that the size of the energy gap is a significant determinant of relative protein folding rates. 


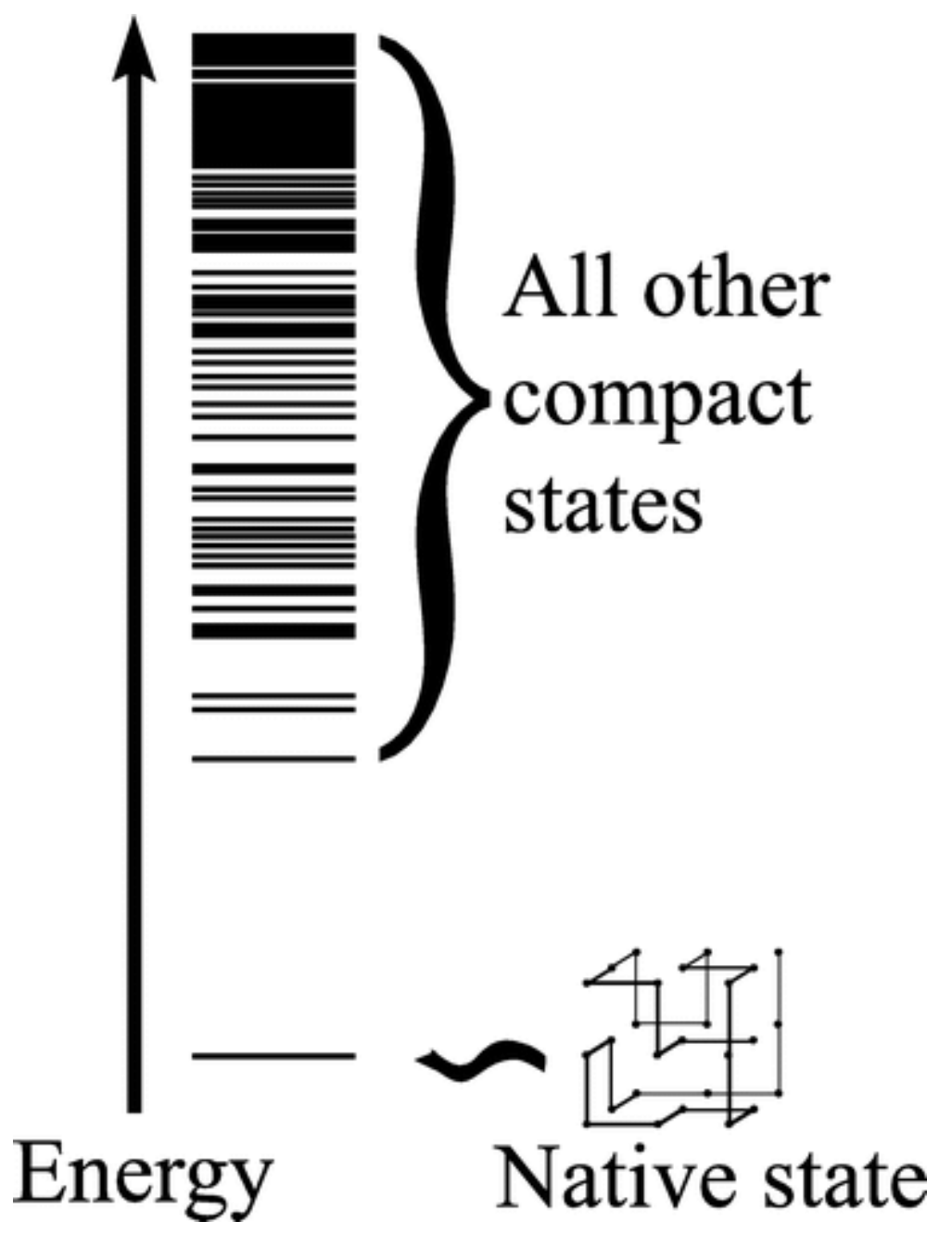

Figure 4 Karplus and coworkers argue that a large gap between the energy of the native state and the second-lowest-energy, maximally compact state is a "necessary and sufficient condition for [rapid] folding" (14) and that the size of this gap is correlated with folding rates (16). 


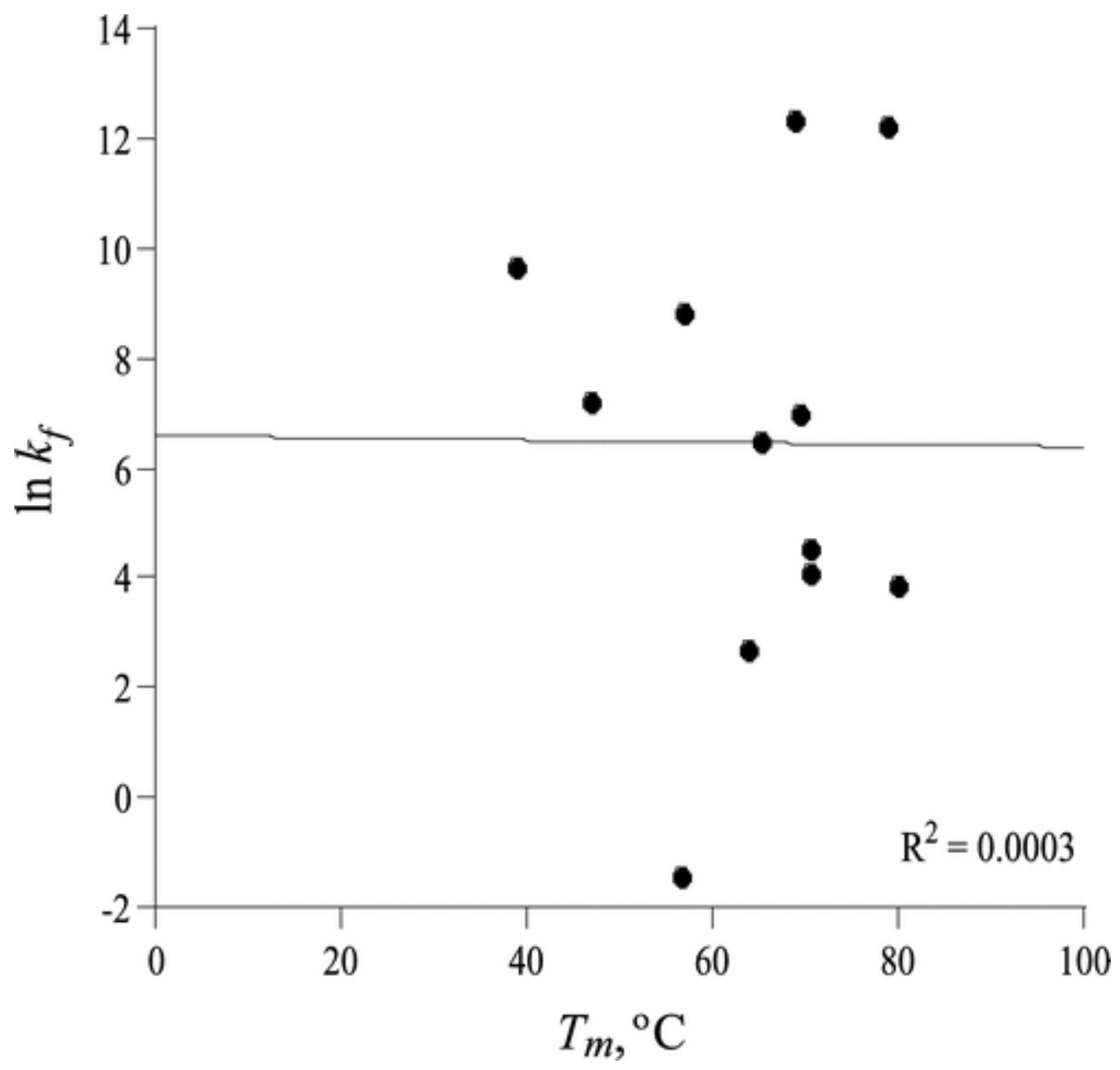

Figure 5 Although it is not possible to test the energy gap hypothesis directly (because the energy gap does not correspond to any experimental observable), an indirect experimental test is provided by the empirical observation that the melting temperatures $\left(T_{m}\right)$ of lattice polymers are highly correlated with both the size of their energy gap and their folding rates (16). No significant correlation between $T m$ and folding rates is observed, however, when a set of unrelated, single-domain proteins is characterized in the laboratory. Shown are data from 12 nonhomologous two-state proteins randomly selected from the literature $(91,93-$ 105).

\section{Collapse Cooperativity}

Thirumalai and coworkers (19-24) have demonstrated that the relative folding rates of many simple on- and off-lattice polymer models are defined by a dimensionless, equilibrium parameter termed $\sigma . \sigma$ is a measure of the ease with which the denatured state undergoes nonspecific, Flory-type coil-to-globule collapse relative to the ease with which the protein folds as temperature or solvent quality (e.g., denaturant concentration) is reduced (Figure 6). Two equivalent measures of $\sigma$ can be derived. The first, $\sigma_{T}$, is defined in terms of thermal unfolding and is related to the melting $\left(T_{m}\right)$ and collapse $\left(T_{\theta}\right)$ temperatures by

$$
\sigma_{T}=1-T_{m} / T_{\theta}, \quad 2 .
$$

where $T_{m}$ is the temperature (in Kelvins) at which half of a population of polymers is folded, and $T_{\theta}$ is the temperature at which the mean dimensions of the ensemble are 
midway between those of the folded and unfolded states. The second, $\sigma_{D}$, is reported to be an equivalent parameter obtained via chemical denaturation experiments (22; D. Thirumalai and D. Klimov, personal communication). It is defined by

$$
\sigma_{D}=1-C_{M} / C_{\theta}, \quad 3 .
$$

where $C_{M}$ is the denaturant concentration at which half of the population of polymers is folded, and $C_{\theta}$ is the denaturant concentration at which the mean dimensions of the ensemble are midway between those of the fully folded and unfolded ensembles. Both definitions of $\sigma$ are thus equilibrium measures of the cooperativity of the collapse of the unfolded state to form the native protein.

Two research groups have explored the relationship between $\sigma$ and the folding kinetics of simplified computational models. Thirumalai and coworkers (24) have demonstrated that on- and off-lattice polymer sequences with high $\sigma$ fold orders of magnitude more slowly than sequences that contract only at conditions nearer the midpoint of the equilibrium folding transition. Karplus and coworkers (16) have also investigated the relationship between $\sigma$ and folding rates. Although these authors report that folding kinetics correlate more strongly with the energy gap criterion than with $\sigma$ (and, indeed, raise questions about the definition of the parameter), they nevertheless observe a statistically significant correlation between $\sigma$ and folding rates across several lattice models. Simulations suggest that six order of magnitude change in folding rate should correspond to changing by $\sigma$ more than $0.5(20,24,25)$, a difference well within reach of experimental verification.

And is $\sigma$ correlated with folding rates in the laboratory? To date $\sigma_{D}$ has been experimentally defined for four simple, single-domain proteins spanning a greater than 30,000-fold range of rates (26). All four proteins exhibit $\sigma_{D}$ effectively indistinguishable from zero (Figure 7). Similarly, we can employ previously reported data on the thermal unfolding of cytochrome $c$ to determine that, for that protein, $\sigma_{D}$ is also $\sim 0$ (27). Thus in keeping with theory, near zero values of $\sigma$ are associated with the rapid folding of naturally occurring, two-state proteins. In contrast to the behavior of lattice and simple off-lattice polymers, however, the relative folding rates of simple proteins are not defined by this equilibrium measure of collapse cooperativity. 


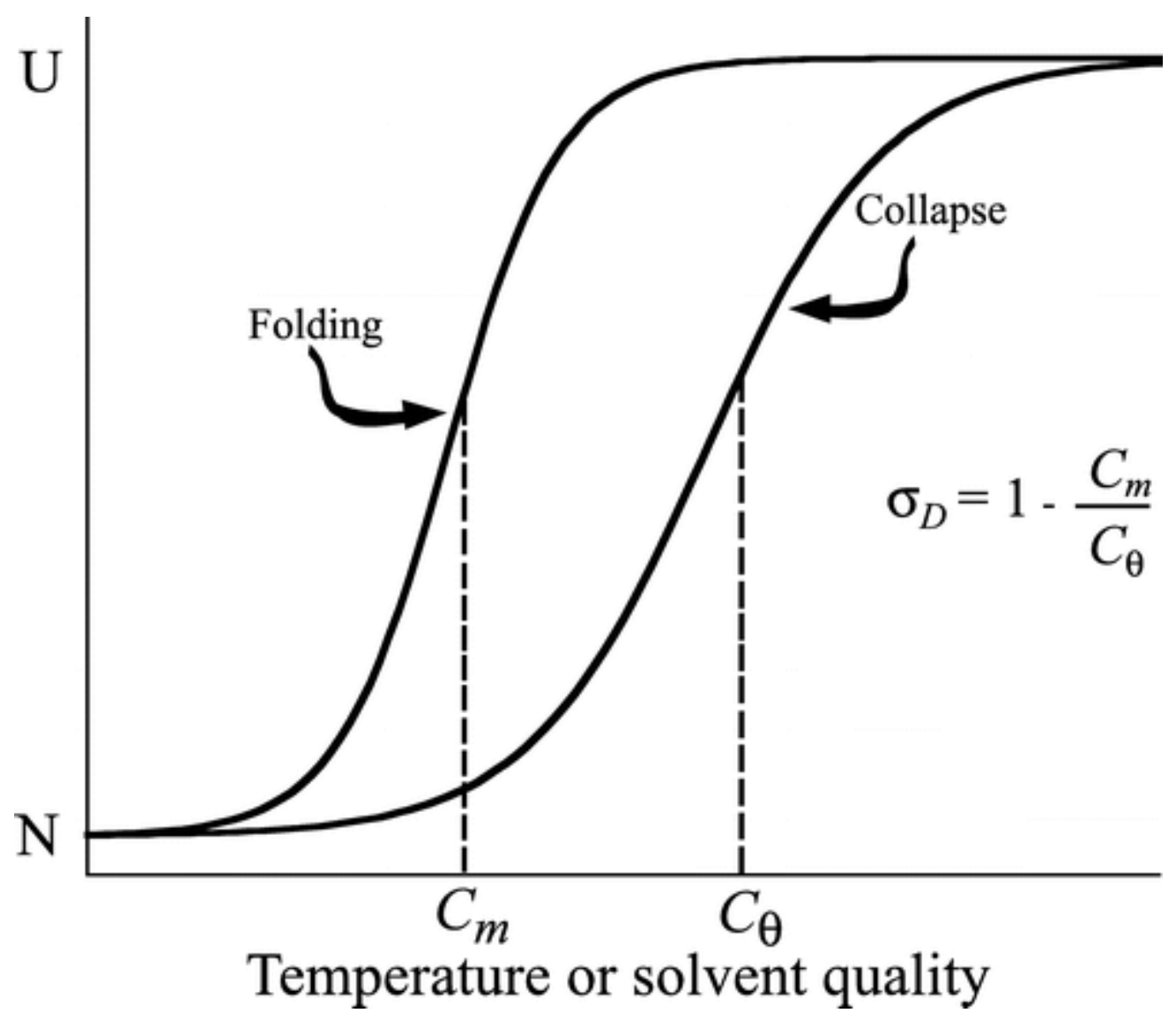

Figure 6 Thirumalai and coworkers (19-24) have reported that the dimensionless, equilibrium collapse parameter $\sigma$ is highly correlated with the relative folding rates of simple on- and off-lattice polymers. $\sigma$ is a measure of the point at which the unfolded polymer undergoes nonspecific coil-to-globule collapse relative to the point at which it folds as the solvent quality or temperature is reduced. 


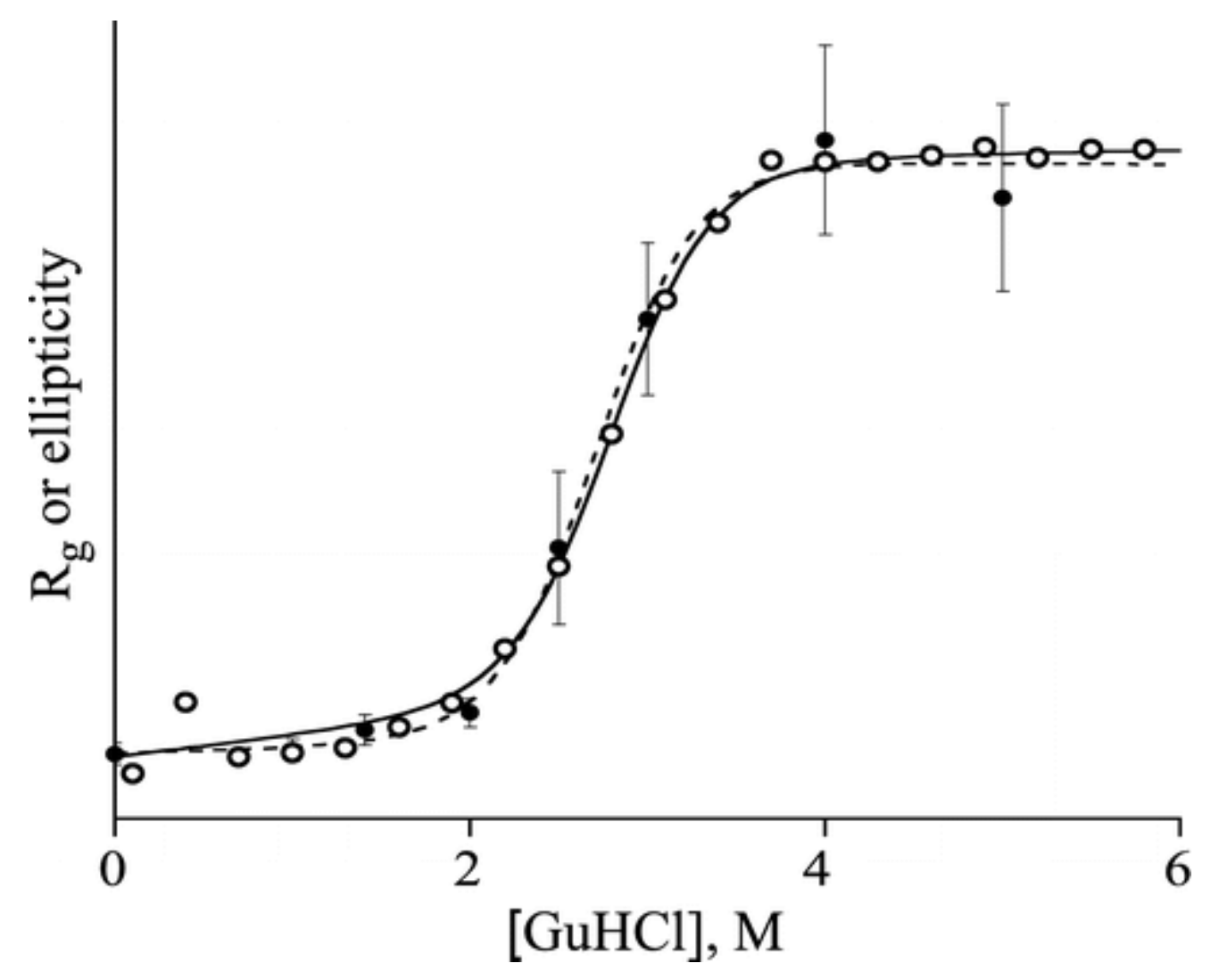

Figure 7 We can measure the putative kinetic determinant $\sigma$ by monitoring collapse via small angle X-ray scattering and folding via near UV-circular dichroism. Shown is the equilibrium folding and collapse of the single-domain protein acyl phosphatase. As for all two-state proteins characterized to date (26), the $\sigma$ of this protein is within error of zero, and thus this parameter is not correlated with relative folding rates in the laboratory.

\section{All Folding Criteria Optimal?}

Experimental studies indicate that the criteria that determine folding rates in simple computational models do not account for the range of folding rates observed for simple, single-domain proteins. Indeed, the energy gaps of nearly all two-state proteins are unmeasurably large and both energetic roughness and $\sigma$ typically adopt the unmeasurably small values that are associated only with the most rapidly folding lattice polymers. Thus, it appears that although biologically relevant folding rates are associated with a smooth landscape/large energy gap/low $\sigma$, differences in these parameters do not contribute significantly to the six order of magnitude range of two-state folding rates observed in the laboratory.

\section{EXPERIMENTALLY MOTIVATED THEORIES OF PROTEIN FOLDING KINETICS}

In contrast to simulation-based theories of protein folding, several theories have arisen directly from the study of experimentally observed folding rates. More precisely, these theories have emerged from studies of how folding rates change with changing solvent conditions, under the influence of mutations, and across nonhomologous proteins. Two of 
these, the nucleation-condensation and topomer search models, specifically address the issue of why some proteins fold more rapidly than others. Here we describe these two models in detail, placing emphasis on their ability to explain or predict the vast range of experimentally observed folding rates.

\section{Nucleation-Condensation Model}

It is well established that native-like interactions are formed in the rate-limiting step of folding. For example, the perfectly exponential denaturant dependencies of folding rates (termed linear chevron behavior) demonstrate that the folding transition state contains interactions similar to those that stabilize the native state [reviewed in (28)]. The role that native interactions play in defining folding rates is further supported by reports that native-state stability is an important determinant of the relative folding rates of topologically similar proteins $(13,29)$ and, often, of point mutants of a single protein (29-32).

Protein engineering studies, termed $\theta$-value analysis (33), have further clarified the nature of this native-like transition-state structure and led to the development of the nucleation-condensation model of folding (Figure 8). This theory is based on the observation that a subset of all mutations destabilize the folding transition state approximately as much as they destabilize the native state, suggesting that the mutated residues are in a near-native environment during the rate-limiting step in folding [reviewed in (34)]. Although these structured transition-state residues are frequently distant in the protein sequence, they often cluster when mapped onto the native fold (33). Taken together, these observations support the hypothesis that folding is akin to nucleation in a phase transition. That is, a necessary and sufficient condition to surmount the rate-limiting step in folding is the formation of a small, specific nucleus of native structure upon which the remaining structure condenses, and differences in the stability of this nucleus contribute to relative folding rates (35).

The evidence in support of the nucleation-condensation model of folding is, however, rather qualitative; although native-like structures are clearly present in the folding transition states of many proteins, their quantitative contribution to relative folding rates has not been established. Indeed, much recent evidence suggests that differences in the stability of the folding nucleus contribute comparatively little to the six order of magnitude range of two-state folding rates. This evidence includes the observation that the vast majority of point mutation [reviewed in (28)] and even much larger sequence changes (36-38) produce less than one order of magnitude changes in folding rates. Similarly, circular permutations $(39,40)$ and covalent circularizations $(41)$ that largely or entirely disrupt the $\theta$-value-defined nucleus produce only rather minor, three- to sevenfold changes in rate (Figure 9). Therefore, though it is extremely well established that a nucleus of native-like structure is formed in the rate-limiting step of folding, it appears that even large-scale alteration of this structure - and thus presumably its thermodynamics - typically fails to change folding rates significantly, suggesting in turn that the precise details of the folding nucleus are not the major determinant of relative folding rates. 


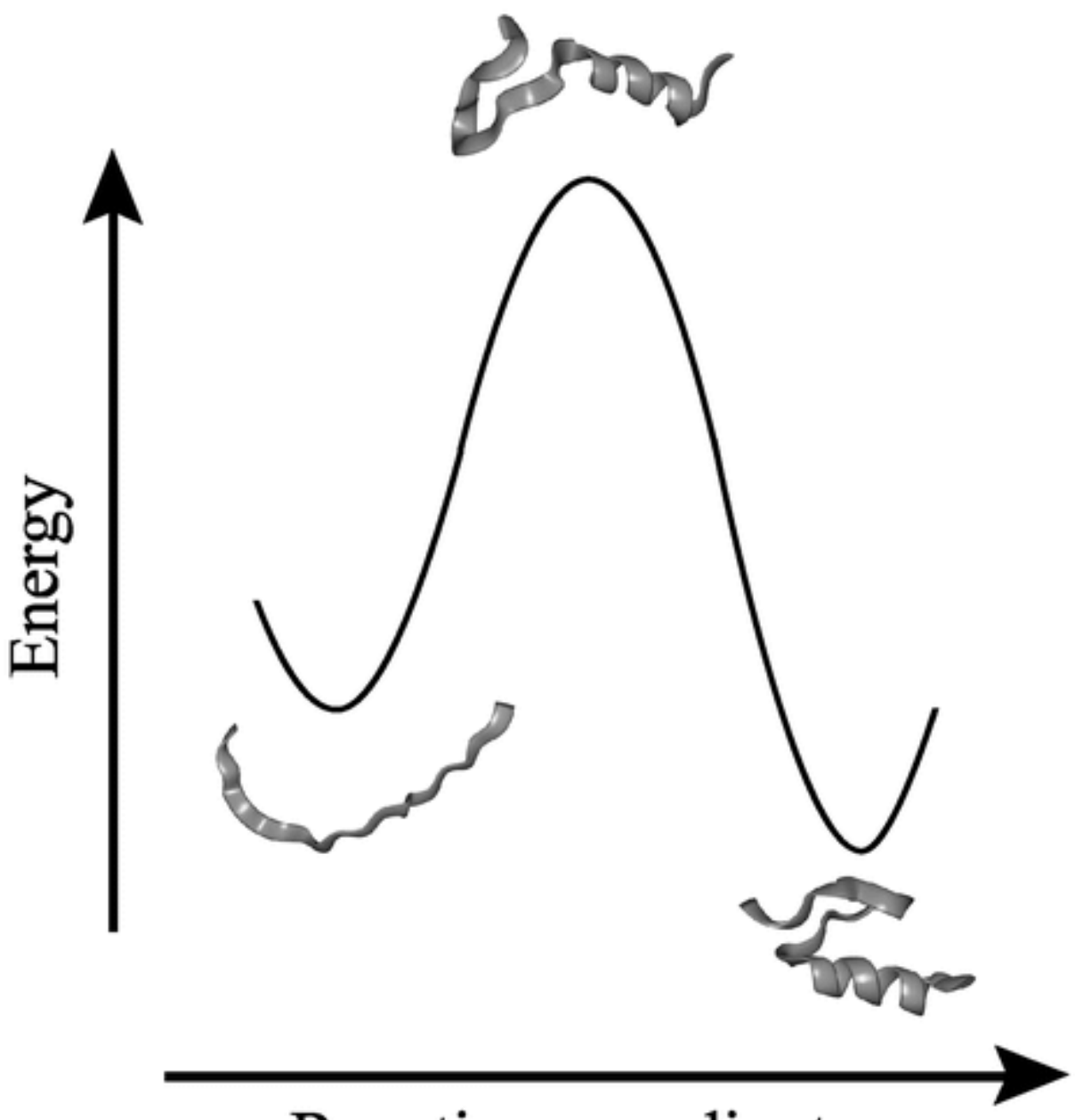

Reaction coordinate

Figure 8 Mutagenesis studies [termed $\theta$-value analysis, reviewed in (34)] and other, less direct experimental evidence suggest that the folding transition state contains a nucleus of native-like structure. This has led to the hypothesis that the formation of this nucleus is a necessary and sufficient condition to ensure that the rate-limiting step in folding has been surmounted and that differences in the stability of the folding nucleus account for differences in folding rates (35). 


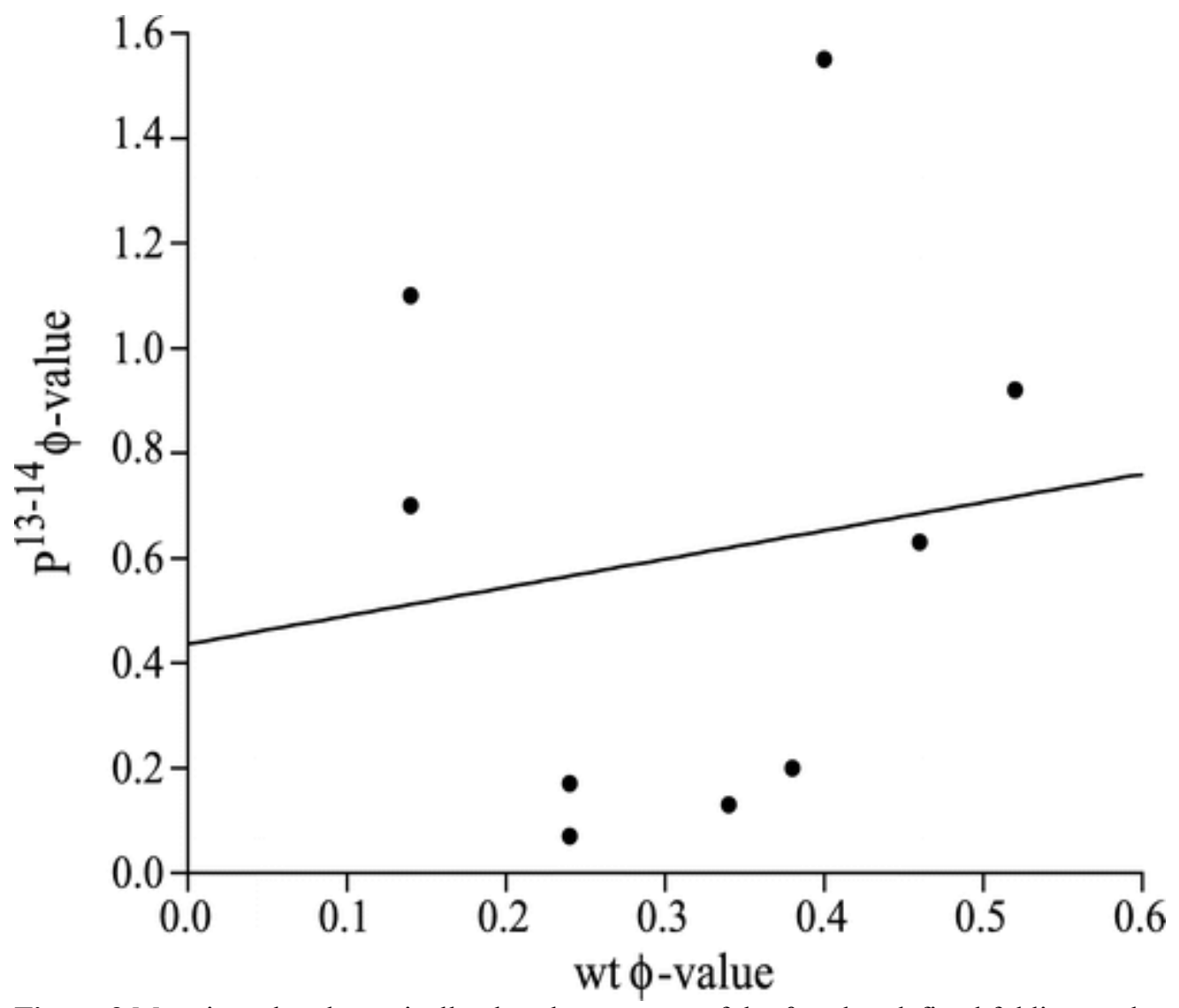

Figure 9 Mutations that dramatically alter the structure of the $\theta$-value-defined folding nucleus typically do not significantly alter folding rates. Shown is the correlation between a residue's $\theta$-value in the wild-type protein S6 and a circularly permuted variant [data adopted from (40)]. The statistically insignificant correlation demonstrates that the mutation entirely disrupts the wild-type nucleus. Despite completely rearranging the folding nucleus, the mutation accelerates the folding rate by a relatively minor factor of $\sim 2$ (40).

\section{Topology as a Determinant of Rates}

In contrast to the suggestion that the thermodynamics of the transition state are dominated by a small subset of native interactions, in the late $1990 \mathrm{~s}$, it was reported that folding rates are strongly correlated with an empirical metric of global native-state topological complexity termed contact order (42). More recently a number of additional, empirical measures of topology have been reported, including the number of sequencedistant contacts per residue (43), the fraction of contacts that are sequence local (35), the total contact distance (44), and linear models of secondary structure content (45). All of these metrics predict folding rates approximately equally accurately, suggesting they reflect a common underlying physics. Because all four metrics reflect empirical observations rather than theoretical models, they do not, however, directly define the mechanistic origins of this physics.

Because contact order was the first topological metric reported to correlate with folding rates, it has received the most attention vis-a'-vis efforts to reconcile the empirically observed topology-rate relationships with a quantitative, mechanistic model of folding. For example, because contact order is related to the sequence separation between contacting residues it has been suggested that it relates to the entropic cost of the 
loop closures required to surmount the rate-limiting step in folding $(42,46-48)$. Unfortunately, however, loop-closure entropy is proportional to the logarithm of loop length rather than loop length per se (49), and the average log (separation) between contacting residues is more poorly correlated with rates than is contact order as originally defined (K.W. Plaxco, unpublished observations). Perhaps because of this, several theoretical models of folding based on balancing loop-closure entropy with favorable side chain interactions exhibit poorer correlations with folding rates than the original, empirical observation itself $(46,47)$. Similarly, relative contact order (the average contact separation in terms of fraction of total peptide length) predicts rates significantly more accurately than absolute measures of the average sequence separation of contacting residues $(50,51)$. This produces the counterintuitive result that, of two proteins with the same average contact separation, the longer protein folds faster. Observations such as these lead inevitably to the possibility that contact order predicts rates not because it is directly related to the underlying mechanism of folding but because it is a proxy for some other physically more reasonable parameter.

The first hint as to what this more mechanistically relevant parameter is came from the work of Goddard and coworkers (52), who related folding times to the number of gross topologies a protein must sample in order to enter the native energy well. In this topomer sampling model, the first stage of folding is dynamic as the unfolded chain diffuses between distinct gross topologies (topomers) (Figure 10, $B$ to $C$ transition). When the native topomer is found, stabilizing interactions can rapidly accrete, trapping the protein in its native conformation (Figure 10, $C$ to $E$ transition). Two properties of simple, singledomain proteins suggest that this type of search could dominate two-state folding rates. First, the formation of local structures, such as helices, hairpins, and loops, is orders of magnitude more rapid than the rate-limiting step in folding [reviewed in (53)]. Second, the folding free energies of such isolated structural elements, indeed, of almost all of the partially folded and misfolded states of single-domain proteins, are near or above zero (54-56). If proteins fold via the diffusional rearrangement of these unstable, rapidly interconverting elements, then the rates will depend on the difficulty of finding the native topomer among all other, incorrect topomers. If all topomers are equally well populated, folding rates (for a given protein length) will depend only on the topomer sampling rate (52). If, in contrast, topologically distinct topomers are not equally well populated, rates will be determined by the topomer search rate, which is a function of both the topomer sampling rate and the fraction of unfolded conformations that are in the native topomer (57-59). The latter model predicts that relative folding rates are proportional to the probability of achieving the native topomer and that differences in the probability of achieving various native topomers could account for the vastly differing folding rates observed for topologically distinct proteins.

The topomer search model provides a rationalization for the wide range of two-state folding rates, but does it quantitatively account for that range? We have recently developed a simple method of estimating the probability of an unfolded chain adopting any given topomer that accurately predicts the folding kinetics of single-domain proteins $(58,59)$. The method emerged from simulations of inert, Gaussian chains, which suggested that the probability of adopting a given topomer is quantifiable via a straightforward approximation arising from two simplifying effects. First, because the locations of residues that are close in sequence are highly correlated, the probability of 
achieving a given topomer is dominated by pairs of residues that are distant in the sequence. The second is that the probability of ordering the chain is well described by a mean-field approximation: Once a sufficient number of sequence-distant pairs of residues are brought into proximity, the probability of each of the remaining ordering events becomes independent of all other ordering events and approximately constant. The probability of forming the native topomer, $P\left(Q_{D}\right)$, is approximated by replacing the unique probability of ordering each specific pair with the average probability of ordering a pair (58):

$$
P\left(Q_{D}\right) \quad \alpha<K>Q_{D},
$$

where $Q D$ is the number of sequence-distant pairs whose proximity defines the topomer, and $\langle K\rangle$ is the average equilibrium constant for residue pairs being in proximity (and is less than unity). Folding rates should, in turn, be proportional $P\left(Q_{D}\right)$.

The prediction that folding rates relate to $Q_{D}$ provides a means of testing the topomer search model. In order to perform this test, however, $Q_{D}$ must be defined. The definition of $Q_{D}$ assumes that any pair of residues separated by more than $l_{c}$ residues along the sequence and within distance $r_{c}$ in the native state will be in proximity in the native topomer. The model is surprisingly insensitive to the precise values of these parameters; over a wide range of $l_{c}$ and $r_{c}, Q_{D}$ is correlated with $\log (\mathrm{kf})$ with a correlation coefficient of $r 2>0.75$ (Figure 11). Thus this simple model captures in excess of $3 / 4$ of the variance in our kinetic data set using only the fitted parameters $\langle K>$ and the proportionality constant.

The predictive value of the topomer search model can be improved by introducing a length dependence; the extent to which a protein's folding rate differs from that predicted by the topomer search model is strongly correlated with chain length (59). This correlation, however, suggests that, for two proteins with the same $Q_{D}$, the longer protein folds more rapidly. This statistically robust observation, which is counter to the predictions of many simulation-based theories $(52,60,61)$, is thought to arise due to crowding stemming from excluded volume and persistence length effects (59). With the addition of this crowding effect via the equation

$$
k_{f} \propto Q_{D} \cdot J^{Q D / N},
$$

where $J$ is a constant $(J<1)$ that is analogous to $\langle K>$, the correlation coefficient for the topomer search model increases to $r 2=0.85$ (59). The ability of this simple, near firstprinciples model of folding kinetics to capture $85 \%$ of the variance in two-state folding rates further supports the hypothesis that the topomer search process dominates relative barrier heights. 


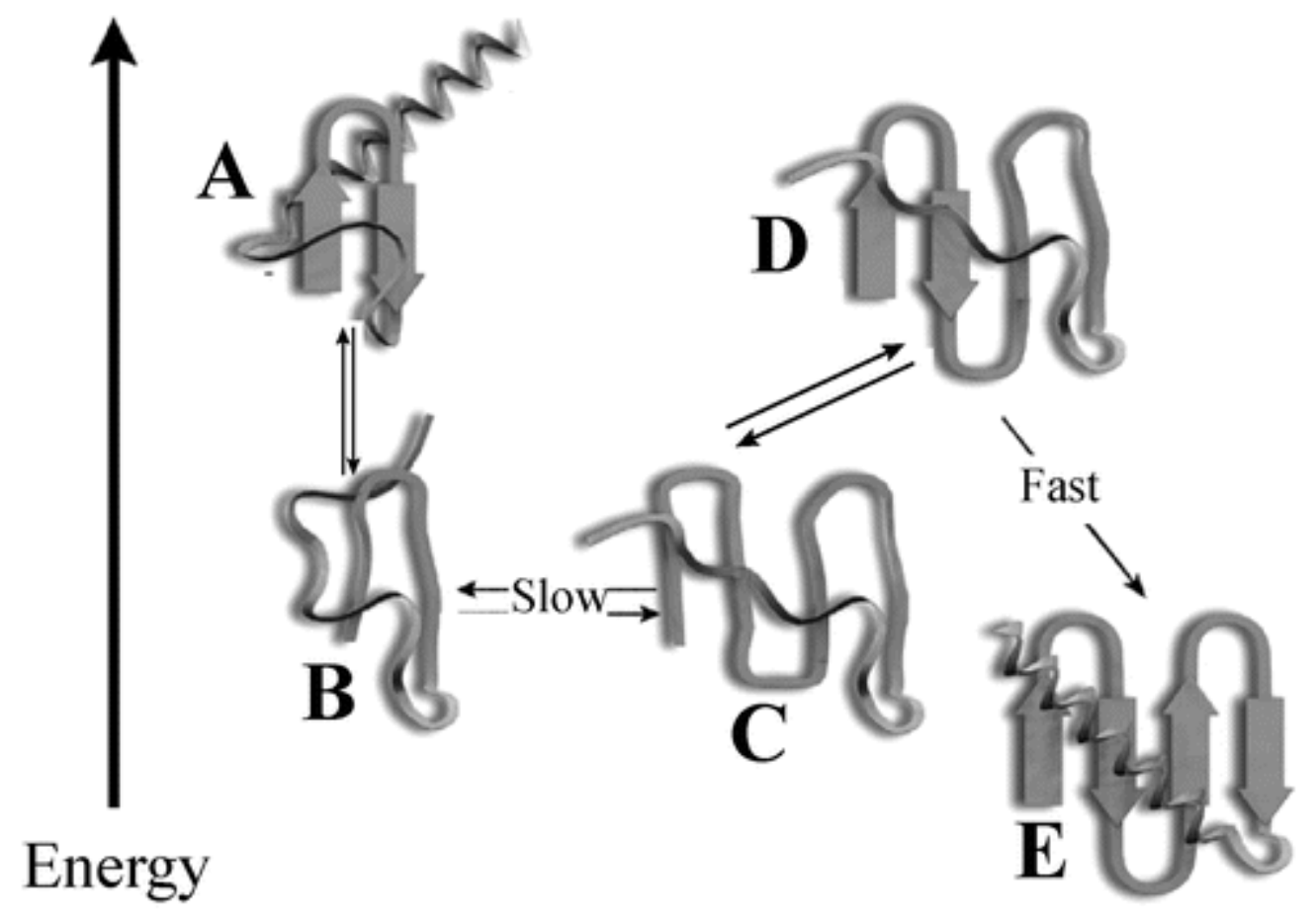

Figure 10 The essence of the topomer search model is that the rate with which an unfolded polymer diffuses between distinct topologies is much slower than the rate with which local structural elements zipper (and, critically, unzip) [reviewed in (59)]. It is well established that the formation of helices, loops, and other sequence-local structural elements (e.g., $B$ to $A$ transition) is significantly faster than the ratelimiting step in folding. Because the free energy of these partially folded states (e.g., $A$ ) is almost invariably $\geq 0$ for two-state proteins, their disruption (e.g., the $A$ to $B$ transition) is more rapid still. Given these constraints, the rate-limiting step in folding might be the slow, large-scale diffusion to find the set of conformations (e.g., $C$ ) close enough to the native topology that they can zipper deeply into the stable, native well $(E)$ without requiring slow, large-scale topological rearrangements. Central to this argument is the suggestion that, while the formation of specific, native-like interactions may be necessary in order to surmount the rate-limiting step $(D)$, they are neither sufficient to ensure folding (e.g., $A$ ) nor a major determinant of relative barrier heights. 


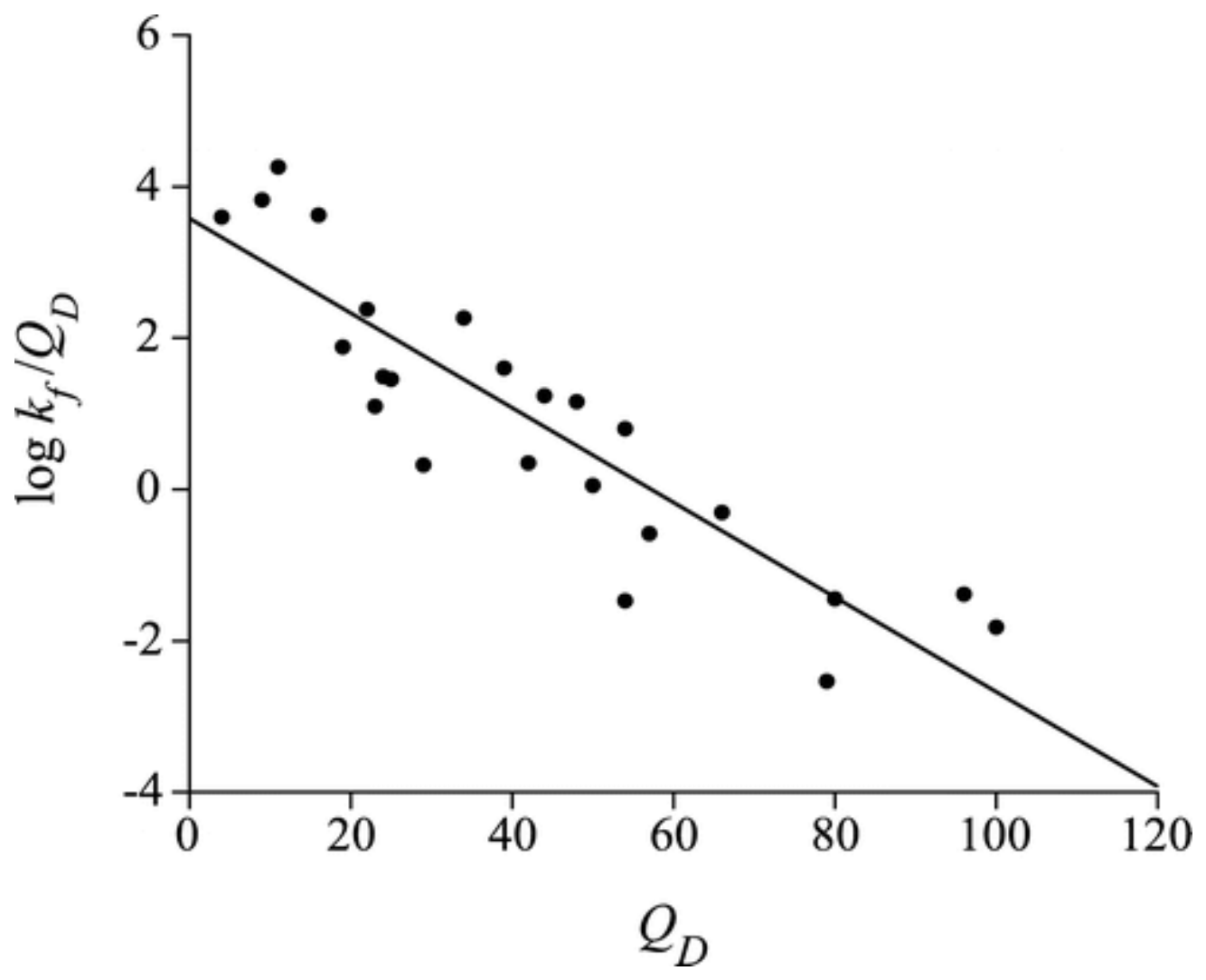

Figure 11 A quantitative version of the topomer search model predicts a simple relationship between folding rates and the number of sequence-distant native contacts $\left(Q_{D}\right)$. As illustrated here, this relationship holds across a dataset of some two-dozen nonhomologous two-state proteins with a correlation coefficient of $r^{2}>0.75$, suggesting that this simple model captures more than $3 / 4$ of the variance in two-state folding rates (59).

\section{A Simple, Predictive Toy Model of Two-State Folding?}

The limiting version of the topomer search model assumes that achieving the native topomer is the rate-limiting step in folding and that side-chain interactions do not contribute significantly to the folding barrier (59). In contrast, the original nucleationcondensation model stipulated that the folding barrier is surmounted when a nucleus of native structure is formed (62), presumably irrespective of the topology of the remainder of the chain. Reality clearly lies somewhere on the spectrum between these two extremes, and both a nucleus of native-like interactions and a grossly native topology are formed in the rate-limiting step in folding $(58,63)$. The observation that the simplest, most extreme version of the topomer search model captures $75 \%$ to $85 \%$ of the variance in two-state folding rates, however, suggests that this toy model of folding may be a reasonable starting point for further advances in theory. We are optimistic that when specific chemical details (e.g., nucleating interactions) are added to the topomer search model, its predictive value will be further improved, and we note that potentially promising advances in a similar direction have already been reported $(64,65)$.

RECONCILING THE BEHAVIORS OF LATTICE POLYMERS AND PROTEINS 
In thinking of the minimal toy model of folding that captures enough of the relevant physics to recapitulate experimentally observed folding behaviors, we come to the question of how and why the folding of lattice polymers differs from that of real proteins. To address these questions, we must consider that simple computational models typically employed in folding simulations differ from proteins in at least two significant aspects. First, while the energy landscapes of most polymer models are usually quite rugged; those of almost all simple, single domain proteins are extremely smooth. Second, in contrast to lattice polymers, for which rates are not defined by "the number of shortversus long-range contacts in the native state" (14), topological effects dominate the relative folding rates of simple, single-domain proteins. Recent experimental and simulations-based studies are providing insights into the origins of these important discrepancies.

\section{The Origins of Optimal Energy Landscapes}

A possible origin of the near-universal observation of smooth landscapes/large energy gaps/low _ among naturally occurring proteins is that these properties are extremely common features of thermodynamically foldable polymers. Lattice polymer simulations suggest, however, that this is not the case. For example, it has been established that lattice polymer sequences with a pronounced energy gap are relatively rare; simulations of randomly selected 27 -mer lattice polymers indicate that only $3 \%$ to $15 \%$ of such sequences encode a sufficiently large energy gap to ensure rapid folding $(14,66,67)$, and the fraction of rapidly folding sequences may be yet smaller for 125-mer lattice systems (68). Near zero $\sigma$, which equates to rapid, two-state folding, is similarly a relatively rare property of randomly selected lattice and simplified off-lattice polymer sequences (24). Thus simulation studies do not appear to support the hypothesis that smooth energy landscapes/large energy gaps/low $\sigma$ are a common property of thermodynamically foldable polymers; we must look elsewhere for reasons underlying the ubiquity of rapid folding among naturally occurring proteins.

Alternatively, smooth energy landscapes/large energy gaps/low $\sigma$ may in fact be rare, but evolutionary pressures aimed specifically at ensuring rapid folding guarantee that naturally occurring proteins are selected from the small subset of sequences that exhibit these critical properties. This hypothesis is supported by studies suggesting that rapidly folding sequences will be produced only rarely in the absence of selective pressures or design constraints aimed at ensuring rapid folding $(69-73,74)$. Experimental tests of this hypothesis, however, are to be found in observations of the folding kinetics of a number of de novo designed proteins. The algorithms by which these molecules have been designed utterly ignore folding kinetics [(75-77); also see the critical commentary in (78)]. Nevertheless, all of the nearly one dozen de novo designed proteins characterized to date fold approximately as fast as or faster than the analogous naturally occurring proteins (38, 79; F. Gai, personal communication; M. Scalley-Kim \& D. Baker, personal communication), suggesting that even in the absence of explicit kinetic selections the large majority of thermodynamically foldable sequences fold rapidly. Rapid folding therefore appears to be relatively common even among sequences that have not been the subject of explicit selection or design aimed at optimizing the energy landscape/energy gap/ $\sigma$. 
The discrepancy in the frequency with which rapid folding arises among protein sequences relative to the paucity of rapidly folding lattice-polymer sequences may be a function of the topologies that natural selection and human design have achieved. Exhaustive simulations demonstrate a close relationship between the native structure of a polymer and the properties of its energy landscape. These simulations suggest that, although most structures are the unique ground state of only a few sequences, a small subset of all structures are encoded by a very large number of sequences (80-83). Naturally occurring protein folds and existing de novo designed proteins are thought to represent such highly designable structures $(80,83)$. Critically, simulation studies indicate that highly designable structures almost invariably exhibit the smooth landscapes and large energy gaps that theory associates with rapid folding $(82,83)$. Though smooth landscapes/large gaps/low $\sigma$ may be rare overall, they may be common among sequences that encode naturally occurring or otherwise designable topologies, thus explaining why, in contrast to randomly selected lattice polymer sequences, energy-landscape issues do not dominate the folding of proteins.

\section{The Origins of Topology-Dependent Rates}

The observation that the large majority of naturally occurring or designed proteins exhibit smooth energy landscapes also provides a potential explanation of why the folding kinetics of simple proteins are dominated by topological constraints, rather than the energy-landscape effects that dominate computational models. Namely, it is possible that subtle topological effects will become apparent only in the absence of potentially more dominant energy-landscape issues. Gō polymers, which have very smooth energy landscapes due to the absence of nonnative stabilizing interactions, provide a means of testing this hypothesis (84). Extensive folding simulations of these systems, however, indicate that smooth energy landscapes alone are not sufficient to generate strongly topology dependent folding rates; on-lattice Gō polymer folding rates are effectively uncorrelated with topology $(85,86)$. The folding rates of more sophisticated, off-lattice Gō polymers likewise exhibit little (87) if any (88) topology dependence. No matter how smooth their energy landscapes, none of the traditional polymer models exhibit the dramatic topology dependence observed in two-state protein folding.

The topomer search model provides a rationale for the lack of topology dependence among even lattice polymers with smooth energy landscapes. The topomer search dominates folding kinetics because the folding of small proteins is extremely cooperative; breaking noncovalent native-state interactions lowers the energetic barrier to the breaking of additional interactions, producing a nonlinear relationship between free energy and the number of native contacts [for an interesting discussion of cooperativity and its relationship to two-state folding, see (89)]. Because of this cooperativity, an excess of $90 \%$ of the native structure is required for the free energy of a typical single-domain protein to drop below zero (54-56). The topomer search dominates rates because this level of cooperativity ensures that only unfolded molecules in the native topomer can zipper sufficient contacts to fold productively, and thus the entropic cost of finding the native topomer is a major contributor to the folding barrier. In comparison to proteins, the folding of traditional Gō polymers is noncooperative (90); the free energy of partially folded Gō lattice polymers is a relatively weak, linear function of the total number of interactions present (85). Gō polymers can, however, be forced to adopt a degree of 
cooperativity by defining the energy of a given conformation as a nonlinear function of the number of interactions present. Consistent with the predictions of the topomer search model, the introduction of such cooperativity leads to a highly significant relationship between topology and lattice-polymer folding rates $(85,86)$. If folding is cooperative and the energy landscape is smooth (i.e., lacking nonnative traps), only those unfolded conformations in the native topomer can fold productively, and for this reason, the entropic cost of the topomer search will dominate relative folding rates.

Simulations thus support the suggestion that the topology-dependent folding rates observed for simple, single-domain proteins are an unavoidable consequence of their highly cooperative folding. In addition to producing topology-dependent kinetics, however, the addition of cooperativity also decelerates the folding of Gō polymers ( 85 , $86,90)$. Does cooperativity also decelerate the folding rates of real proteins? In net it may not; we have speculated that the net effect of cooperativity is to actually accelerate folding by destabilizing partially structured, misfolded states relative to the native fold (smoothing the landscape/increasing the gap/lowering $\sigma$ ) to a greater extent than it decelerates folding by destabilizing potentially productive intermediates (85). This in turn suggests that the observed dominance of topology in defining folding rates is a consequence of the cooperativity necessary to ensure the formation of an energy landscape that can support rapid folding.

\section{CONCLUSIONS}

The criteria that distinguish rapidly folding computational models from those that fold more slowly do not account for the broad range of rates observed for the folding of twostate proteins; the experimentally measurable criteria associated with rapidly folding lattice and off-lattice computational models appear to be perfectly optimized for many naturally occurring proteins. The rapid folding of de novo designed proteins also suggests that this optimization does not require explicitly kinetic selective pressures but is associated with designability. And although native-like interactions are clearly formed during the rate-limiting step in folding, they are apparently not a dominant contributor to relative barrier heights. Finally, a simple mathematical description of the diffusive search for the correct overall topology, the topomer search model, accurately predicts relative folding rates. We believe that the topomer search dominates folding of two-state proteins because their equilibrium folding is so cooperative that only unfolded conformations in the native topomer can zipper sufficient structure for the free energy to drop below zero, and thus the entropic cost of finding the native topomer is a major contributor to the folding barrier. We speculate that this cooperativity provides a means of destabilizing partially structured, misfolded states relative to the native fold, thereby accelerating folding rates more by destabilizing traps (smoothing the landscape/improving the energy gap/decreasing $\sigma$ ) than it decelerates them by destabilizing potentially productive intermediates. This suggests that the observed dominance of topology in defining folding rates is a consequence of the cooperativity necessary to ensure the formation of an energy landscape that can support rapid folding.

\section{ACKNOWLEDGMENTS}


In addition to thanking David Baker, Michelle Scalley-Kim, Alan Fersht, and Feng Gai for communicating important unpublished results, the authors wish to thank our many collaborators over the years without whom our contributions to the above described research would not have been possible. Portions of this work were funded by the NIH (R01GM62868-01A1), BioSTAR (s97-79) and an ACS junior postdoctoral research fellowship (ACS CD INC 2-5-00) to BG.

\section{The Annual Review of Biochemistry is online at http://biochem.annualreviews.org}

\section{LITERATURE CITED}

1. Jackson SE. 1998. Fold. Des. 3:R81-91

2. van Nuland NAJ, Chiti F, Taddei N, Raugei G, Ramponi G, et al. 1998. J. Mol. Biol. 283:883-91

3. Wittung-Stafshede P, Lee JC, Winkler JR, Gray HB. 1999. Proc. Natl. Acad. Sci. USA 96:6587-90

4. Zagrovic B, Snow CD, Shirts MR, Pande VS. 2002. J. Mol. Biol. 323:927-37

5. Bryngelson JD, Wolynes PG. 1987. Proc. Natl. Acad. Sci. USA 84:7524-28

6. Onuchic JN, Wolynes PG, Luthey- Schulten Z, Socci ND. 1995. Proc. Natl. Acad. Sci. USA 92:3626-30

7. Thirumalai D, Ashwin V, Bhattacharjee JK. 1996. Phys. Rev. Lett. 77:5385-88

8. Nymeyer H, Garci'a AE, Onuchic JN. 1998. Proc. Natl. Acad. Sci. USA 95: 5921-28

9. Onuchic JN, Luthey-Schulten Z, Wolynes PG. 1997. Annu. Rev. Phys. Chem. 48:545600

10. Socci ND, Onuchic JN, Wolynes PG. 1998. Proteins: Struct. Funct. Genet. 32: 13658

11. Sabelko J, Ervin J, Gruebele M. 1999. Proc. Natl. Acad. Sci. USA 96:6031-36

12. Gillespie B, Plaxco KW. 2000. Proc. Natl. Acad. Sci. USA 97:12014-19

13. Guijarro JI, Morton CJ, Plaxco KW, Campbell ID, Dobson CM. 1998. J. Mol. Biol. 275:657-67

14. Sali A, Shacknovich E, Karplus M. 1994. Nature 369:248-51 
15. Gutin AM, Abkevich VI, Shakhnovich EI. 1995. Proc. Natl. Acad. Sci. USA 92: 1282-86

16. Dinner AR, Abkevich V, Shakhnovich E, Karplus M. 1999. Proteins Struct. Funct. Genet. 35:34-40

17. Mayor U, Johnson CM, Daggett V, Fersht AR. 2000. Proc. Natl. Acad. Sci. USA 97:13518-22

18. Perl D, Welker C, Schindler T, Schroder K, Marahiel MA, et al. 1998. Nat. Struct. Biol. 5:229-35

19. Camacho CJ, Thirumalai D. 1996. Europhys. Lett. 35:627-32

20. Klimov DK, Thirumalai D. 1996. Phys. Rev. Lett. 76:4070-73

21. Klimov DK, Thirumalai D. 1996. Proteins Struct. Funct. Genet. 26:411-41

22. Klimov DK, Thirumalai D. 1998. Fold. Des. 3:127-39

23. Klimov DK, Thirumalai D. 1998. J. Chem. Phys. 109:4119-25

24. Thirumalai D, Klimov DK. 1999. Curr. Opin. Struct. Biol. 9:197-207

25. Veitshans T, Klimov D, Thirumalai D. 1996. Fold. Des. 2:1-22

26. Millet IS, Townsley L, Chiti F, Doniach S, Plaxco KW. 2002. Biochemistry 41: 32125

27. Hagihara Y, Hoshino M, Hamada D, Kataoka M, Goto Y. 1998. Fold. Des. 3:195201

28. Plaxco KW, Simons KT, Ruczinski I, Baker D. 2000. Biochemistry 39:11177-83

29. Clarke J, Cota E, Fowler SB, Hamill SJ.1999. Struct. Fold. Des. 7:1145-53

30. Riddle DS, Grantcharova VP, Santiago JV, Alm E, Ruczinski I, et al. 1999. Nat. Struct. Biol. 6:1016-24

31. Villegas V, Martinez JC, Aviles FX, Serrano L. 1998. J. Mol. Biol. 6:1027-36

32. Martinez JC, Serrano L. 1999. Nat. Struct. Biol. 6:1010-16

33. Jackson SE, elMasry N, Fersht AR. 1993. Biochemistry 32:11270-78

34. Fersht AR. 1997. Curr. Opin. Struct. Biol. 7:3-9 
35. Mirny L, Shakhnovich E. 2001. Annu. Rev. Biophys. Biomol. Struct. 30:361-66

36. Riddle DS, Santiago JV, Bray-Hall ST, Doshi N, Grantcharova VP, et al. 1997. Nat. Struct. Biol. 4:805-9

37. Kim DE, Gu HD, Baker D. 1998. Proc. Natl. Acad. Sci. USA 95:4982-86

38. Gillespie B, Vu D, Shah PS, Marshall S, Dyer RB, et al. 2003. J. Mol. Biol. 330:81319

39. Viguera AR, Serrano L, Wilmanns M. 1996. Nat. Struct. Biol. 3:874-80

40. Lindberg M, Tangrot J, Oliveberg M. 2002. Nat. Struct. Biol. 9:818-22

41. Grantcharova VP, Baker D. 2001. J. Mol. Biol. 306:555-63

42. Plaxco KW, Simons KT, Baker D. 1998. J. Mol. Biol. 277:985-94

43. Gromiha MM, Selvaraj S. 2001. J. Mol. Biol. 310:27-32

44. Zhou HY, Zhou YQ. 2002. Biophys. J. 82:458-63

45. Gong HP, Isom DG, Srinivasan R, Rose GD. 2003. J. Mol. Biol. 327:1149-54

46. Alm E, Baker D. 1999. Proc. Natl. Acad. Sci. USA 96:11305-10

47. Muñoz V, Eaton WA. 1999. Proc. Natl. Acad. Sci. USA 96:11311-16

48. Fersht AR. 2000. Proc. Natl. Acad. Sci. USA 97:1525-29

49. Jacobson H, Stockmayer WH. 1950. J. Chem. Phys. 18:1600-6

50. Grantcharova VP, Alm EJ, Baker D, Horowitz AL. 2001. Curr. Opin. Struct. Biol. $11: 70-82$

51. Ivankov DN, Garbuzynskiy SO, Alm E, Plaxco KW, Baker D, Finkelstein AV. 2003. Protein Sci. 12:2057-62

52. Debe DA, Carlson MJ, Goddard WA. 1999. Proc. Natl. Acad. Sci. USA 96:2596-601

53. Eaton WA, Munoz V, Hagen SJ, Jas GS,Lapidus LJ, et al. 2000. Annu. Rev. Biophys. Biomol. Struct. 29:327-59

54. Flanagan JM, Kataoka M, Shortle D, Engelman DM. 1992. Proc. Natl. Acad. Sci. USA 89:748-52 
55. Ladurner AG, Itzhaki LS, Gay GD, Fersht AR. 1997. J. Mol. Biol. 273:317-29

56. Camarero JA, Fushman D, Sato S, Giriat I, Cowburn D, et al. 2001. J. Mol. Biol. 308:1045-62

57. Debe DA, Goddard WA. 1999. J. Mol. Biol. 294:619-25

58. Makarov DE, Keller CA, Plaxco KW, Metiu H. 2002. Proc. Natl. Acad. Sci. USA 99:3535-39

59. Makarov DE, Plaxco KW. 2003. Protein Sci. 12:17-26

60. Gutin AM, Abkevich VI, Shakhnovich EI. 1996. Phys. Rev. Lett. 77:5433-36

61. Li MS, Klimov DK, Thirumalai D. 2002. J. Phys. Chem. B 106:8302-5

62. Fersht AR. 1995. Proc. Natl. Acad. Sci. USA 92:10869-73

63. Vendruscolo M, Paci E, Dobson CM, Karplus M. 2001. Nature 409:641-45

64. Myers JK, Oas TG. 2001. Nat. Struct. Biol. 8:552-58

65. Islam SA, Karplus M, Weaver DL. 2002. J. Mol. Biol. 318:199-215

66. Shakhnovich E, Farztdinov G, Gutin AM, Karplus M. 1991. Phys. Rev. Lett. 67:1665-67

67. Sali A, Shakhnovich E, Karplus M. 1994. J. Mol. Biol. 235:1614-36

68. Dinner AR, So S-S, Karplus M. 1998. Proteins Struct. Funct. Genet. 33:177-201

69. Guo Z, Thirumalai D. 1996. J. Mol. Biol. 263:323-43

70. Mirny LA, Abkevich VI, Shakhnovich EI. 1998. Proc. Natl. Acad. Sci. USA 95: 4976-81

71. Gutin AM, Abkevich VI, Shakhnovich EI. 1998. Fold. Des. 3:183-94

72. Li L, Mirny LA, Shakhnovich EI. 2000. Nat. Struct. Biol. 7:336-42

73. Betancourt M, Thirumalai D. 2002. J. Phys. Chem. B 106:599-609

74. Larson S, Ruczinski I, Davidson AR, Baker D, Plaxco KW. 2002. J. Mol. Biol. 316:225-33 
75. Dahiyat BI, Mayo SL. 1996. Protein Sci. 5:895-903

76. Walsh STR, Cheng H, Bryson JW, Roder H, DeGrado WF. 1999. Proc. Natl. Acad. Sci. USA 96:5486-91

77. Dantas G, Kuhlman B, Callender D, Wong M, Baker D. 2003. J. Mol. Biol. 332:44960

78. Jin W, Kambara O, Sasakawa H, Tamura A, Takada S. 2003. Structure 11:581-90

79. Hill RB, Bracken C, DeGrado WF, Palmer AGI. 2000. J. Am. Chem. Soc. 122:1161019

80. Govindarajan S, Goldstein RA. 1995. Biopolymers 36:43-51

81. Li H, Helling R, Tang C, Wingreen N. 1996. Science 273:666-69

82. Buchler NEG, Goldstein RA. 2000. J. Chem. Phys. 112:2533-47

83. Miller J, Zeng C, Wingreen NS, Tang C. 2002. Proteins Struct. Funct. Genet. 47: $506-12$

84. Abe H, Go_ N. 1981. Biopolymers 20: 10113-31

85. Jewett AI, Pande VS, Plaxco KW. 2003. J. Mol. Biol. 326:247-53

86. Kaya H, Chan HS. 2003. Proteins 52:524-33

87. Koga N, Takada S. 2001. J. Mol. Biol. 313:171-80

88. Cieplak M, Hoang TX. 2002. Biophys. J. 84:475-88

89. Kaya H, Chan HS. 2002. J. Mol. Biol. 315:899-909

90. Eastwood MP, Wolynes PG. 2001. J. Chem. Phys. 114:4702-16

91. Filimonov VV, Azuaga AI, Viguera AR, Serrano L, Mateo PL. 1999. Biophys. Chem. 77:195-208

92. Deleted in proof

93. Chiti F, van Nuland NAJ, Taddei N, Magherini F, Stefani M, et al. 1998. Biochemistry 37:1447-55

94. Wittung-Stafshede P. 1999. Biochim. Biophys. Acta 1432:401-5 
95. Huang G, Oas TG. 1995. Proc. Natl. Acad. Sci. USA 92:6878-82

96. Huang G, Oas TG. 1996. Biochemistry 35:6173-80

97. Jacob M, Geeves M, Holtermann G, Schmid FX. 1999. Nat. Struct. Biol. 6: 923-26

98. Jackson SE, Fersht A. 1991. Biochemistry 30:10428-35

99. van Nuland NAJ, Meijberg W, Warner J, Forge V, Scheek RM, et al. 1998.

Biochemistry 37:622-37

100. Horng JC, Moroz V, Raleigh DP. 2003. J. Mol. Biol. 326:1261-70

101. Scalley M, Baker D. 1997. Proc. Natl. Acad. Sci. USA 94:10637-40

102. Spector S, Raleigh DP. 1999. J. Mol. Biol. 293:763-68

103. Knapp S, Karshikoff A, Berndt KD, Christova P, Atanasov B, et al. 1996. J. Mol. Biol. 264:1132-44

104. Kubelka J, Eaton WA, Hofrichter J. 2003. J. Mol. Biol. 329:625-30

105. Lindberg MO, Tangrot J, Otzen DE, Dolgikh DA, Finkelstein AV, et al. 2001. J. Mol. Biol. 314:891-900 